\title{
GESTÃO EM ALIMENTOS E BEBIDAS: INDICADORES PARA UM NOVO CAM- PO DE ESTUDOS NO BRASIL
}

\author{
1 Carlos Eduardo de Mattos \\ 2 Mara Lucia de Moura Pontes \\ ${ }^{3}$ Marcio Luiz Marietto
}

\section{RESUMO}

A partir de dezembro de 2015 a Capes reconheceu formalmente a proposta de abertura de um novo curso em Mestrado Profissional em Administração com foco na Gestão em Alimentos e Bebidas. Ainda que no exterior esse campo de pesquisa (Food and Beverage Management) já esteja consolidado, no Brasil ele é formalmente inédito. Este estudo tem o propósito de identificar, por meio de pesquisa bibliográfica nas bases de dados EBSCO e Food and Beverage Management Commons, as áreas de estudo de alimentos e bebidas no campo da administração de empresas. A proposta é organizar as subáreas da administração de empresas que enquadraram, de forma longitudinal, as pesquisas no setor de alimentos e bebidas por meio da quantificação e categorização sistemática dos estudos. Após analisar título, resumo, palavras-chave, introdução e método de 1138 artigos encontrados, foi obtida uma amostra final de 368 estudos relacionados às áreas da gestão em alimentos e bebidas. Os resultados identificaram 18 áreas da administração de empresas em que existem pesquisas sobre alimentos e bebidas. Os resultados também quantificaram o número de publicações e demonstraram, ao longo do tempo, a partir da década de 1960, o progresso e os turns (períodos de grandes evoluções na produção) dos estudos em alimentos e bebidas em cada década. Este artigo contribuiu, de forma seminal, para mapear a área e fornecer indicadores de sua produção científica. $\mathrm{O}$ estudo contribuiu, também, para direcionar investigações futuras.

Palavras-chave: Gestão em Alimentos e Bebidas; Pesquisa Bibliográfica; Produção Científica.

\footnotetext{
${ }^{1}$ Mestrando em Administração de Empresas com foco em Gestão em Alimentos e Bebidas pela Universidade Anhembi Morumbi - UAM, São Paulo, (Brasil). Professor na Disciplina de Gestão de Operações de Comércio Exterior pelo Serviço Nacional de Aprendizagem Comercial - São Paulo, SENAC, Brasil. E-mail: ce.mattos@terra.com.br

${ }^{2}$ Bacharelado em Publicidade e Propaganda pela Faculdade Cásper Líbero, São Paulo, (Brasil). Professora pelo Centro Universitário das Faculdades Metropolitanas Unidas - FMU, São Paulo, Brasil. E-mail: maralucia.m.pontes@gamil.com

${ }^{3}$ Doutor em Administração pela Universidade Nove de Julho - UNINOVE, São Paulo, (Brasil). Docente pela Universidade Anhembi Morumbi - UAM, São Paulo. E-mail: mlmarietto@uol.com.br
} 
Gestão em Alimentos e Bebidas: Indicadores para um Novo Campo de Estudos no Brasil

\title{
FOOD AND BEVERAGE MANAGEMENT: INDICATORS FOR A NEW FIELD OF STUDI- ES IN BRAZIL
}

\begin{abstract}
Since December, 2015, CAPES has been considering the proposal to open a new course in Professional Master in Business Administration with a focus on Food and Beverage Management. Although this research field (Food and Beverage Management) is already consolidated abroad, in Brazil it is formally unprecedented. This study aimed to identify, through bibliographic research on EBSCO and Food and Beverage Management Commons databases, food and beverage study areas in the field of business administration. This research also aims to identify and quantify the maount of studies by the area. The proposal is to organize the subareas of business administration which framed, longitudinally, research in the food and beverage industry by systematic categorization of the studies. After analyzing title, abstract, keywords, introduction and method of 1138 papers, we obtained a final sample of 368 studies in areas of management in food and beverage. The results have identified 18 areas of business administration in which there is research on food and beverage. The results also have quantified the number of publications and demonstrated, over time, from the 1960s, the progress and turns of the studies on food and beverage in each decade. This paper has contributed to map business areas and provide indicators for scientific production. The study also contributes to point out future research.
\end{abstract}

Keywords: Food and Beverage Management; Bibliographic Research; Scientific Production.

\section{GESTIÓN EN ALIMENTOS Y BEBIDAS: INDICADORES PARA UN NUEVO CAMPO DE ESTUDIOS EN BRASIL}

\section{RESUMEN}

A partir de Diciembre de 2015 la CAPES reconoció formalmente la propuesta de abertura de un nuevo curso Máster Profesional en Administración con foco en Gestión de Alimentos y Bebidas. Mismo que en el exterior este campo de pesquisa (Food and Beverage Management) ya este consolidado, en Brasil es formalmente inédito. Este estudio tiene el propósito de identificar, por medio de pesquisa bibliográfica basándose en datos EBSCO y Food and Beverage Management Commons, las áreas de estudio de alimentos y bebidas en el campo de administración de empresas. La propuesta es de organizar las subareas de administración de empresas que encuadraron, de forma longitudinal, las pesquisas del sector de alimentos y bebidas por medio de la cuantificación y caracterización sistemática de los estudios. Después de analizar título, resumen, palabra llave, introducción y método de 1338 artículos encontrados, se obtuvo una muestra final de 368 estudios relacionados a las áreas de gestión en alimentos y bebidas. Los resultados identificaron 18 áreas de la administración de empresas en que existen pesquisas sobre alimentos y bebidas. Los resultados, también, cuantificaron el número de publicaciones y demostraron, al largo del tiempo, a partir de la década de 1960, el progreso y los turns (períodos de grandes evoluciones en la producción) de los estudios en alimentos y bebidas en cada década. Este artículo contribuyo, de forma seminal, para mapear el área y ofrecer indicadores de su producción científica. El estudio contribuyó también para direccionar investigaciones futuras.

Palabras llaves: Gestión en alimentos y bebidas; Pesquisa bibliográfica; Producción científica. 


\section{INTRODUÇÃO}

A Gestão em Alimentos e Bebidas (Food and Beverage Management) é considerada um dos pilares da Gestão da Hospitalidade. Além disso, é considerada uma das disciplinas mais importantes nos cursos de Hospitalidade. Os estudos nesse campo de pesquisa já se mostram consolidados no exterior, devido ao aumento na lucratividade do setor de serviço em alimentos e bebidas. Contudo, no Brasil esse campo de pesquisa ainda é formalmente inédito. Todos os dias, as pessoas ao redor do mundo compram e consomem alimentos enquanto desempenham suas atividades cotidianas. Com a melhora do padrão de vida, as pessoas passam a gastar mais em lazer, incluindo a procura por sabores e experiências diferentes quando buscam a alimentação fora de casa (TEWS \& HOOF, 2011; BARROWS, 2008, p.422; WOOD, 2007).

No Brasil, a Coordenação de Aperfeiçoamento de Pessoal de Nível Superior - Capes autorizou em dezembro de 2015 o programa de Mestrado Profissional em Administração de Empresas com ênfase na Gestão em Alimentos e Bebidas da Universidade Anhembi Morumbi. O programa é inédito no país e tem o objetivo de contribuir para o desenvolvimento de projetos que aprimorem o entendimento de processos e a aplicação de estratégias de gestão no setor de alimentos e bebidas. Além disso, o programa visa identificar competências que proporcionem uma melhoria para as organizações desse setor. Para isso, o curso apoia-se em duas linhas de pesquisa: Negócios em A\&B (L1) e Operações em A\&B (L2). Conforme disponível na página do programa de mestrado da universidade, os objetivos dos principais projetos que norteiam esse programa são: descrever o setor de A\&B e esquematizar sua cadeia produtiva; levantar e classificar as empresas de serviços de restaurantes; compreender a mobilidade corporativa; identificar as melhores práticas de gestão de pessoas no segmento de restaurantes; identificar e mo- delar as diversas práticas de monitoramento no atendimento aos clientes nos restaurantes; e observar os princípios do controle higiênico-sanitário nos restaurantes da cidade de São Paulo (Anhembi Morumbi/Mestrado Profissional, 2016).

Este estudo visou contribuir para um maior esclarecimento da área de gestão em alimentos e bebidas por meio de uma pesquisa bibliográfica nas publicações disponíveis em duas bases de dados distintas: EBSCO e Food and Beverage Management Commons. Uma pesquisa bibliográfica é um método composto por um conjunto de procedimentos que são aplicados exclusivamente sobre fontes bibliográficas, como livros acadêmicos, handbooks, artigos científicos, dissertações, teses, entre outras fontes científicas. Seu principal objetivo é avaliar as tendências sobre um determinado assunto, teoria, disciplina ou campo de estudo, ao longo do tempo (MOTA, FERREIRA, WADA \& SANTOS, 2014; LIMA \& MIOTO, 2007). A busca nessas bases encontrou um total de 1321 artigos, que, após serem organizados, classificados e analisados, foram reduzidos a uma amostra de 368 artigos correlatos com o tema da pesquisa.

Os resultados mostraram um aumento exponencial na produção de estudos relacionados às áreas de gestão desde a década de 1960, quando foram identificados os primeiros artigos. O período pesquisado apresenta três turns em relação à quantidade de publicações. $\mathrm{O}$ primeiro salto aconteceu na década de 1980 , o segundo na década de 2000 e, por fim, o terceiro na década de 2010. O total de publicações nesses três decênios apresentou um número maior que o dobro da soma dos decênios anteriores. Foram identificados, ainda, no mesmo período, 18 áreas de gestão e 21 objetos pesquisados nos estudos selecionados para este trabalho.

Este artigo está estruturado em quatro partes, sendo a primeira delas uma revi- 
são da literatura sobre Gestão em Alimentos e Bebidas e sobre o método Pesquisa Bibliográfica. Na segunda parte é descrito um detalhamento do método utilizado no estudo. Em seguida, são apresentados os resultados da pesquisa, em que gráficos e tabelas ilustram as métricas retiradas das análises das publicações encontradas. E, por fim, foram tecidas as considerações finais acerca dos resultados do estudo.

\section{GESTÃO EM ALIMENTOS E BEBI- DAS}

O fornecimento de alimentos e bebidas fora do lar é uma das mais importantes atividades do setor da hospitalidade e, consequentemente, representa uma parte substancial da economia. O setor de alimentos e bebidas, que começou muito fragmentado, hoje é composto por uma ampla rede de provedores, fornecedores, clientes e outros associados que se relacionam internacionalmente, diminuindo as barreiras e fronteiras desse setor. Os estabelecimentos de alimentos e bebidas vão desde empresas públicas e privadas, passando pela gama de pequenas unidades, operadas por proprietários independentes, grandes corporações multinacionais que gerenciam marcas globais, até caterings que atendem a prisões e luxuosos hotéis. Devido a essa diversidade, percebe-se a dificuldade de se considerar estatísticas consistentes referentes ao setor da hospitalidade e às operações de alimentos e bebidas, pois, como não existe uma definição única quanto às fronteiras dos setores e subsetores desse segmento, não é possível determinar o que deveria ou não ser incluído nessa categoria (DAVIS, LOCKWOOD, ALCOTT \& PANTELIDIS, 2012; BARROWS，2008, p.421).

Davis et al. (2012, p.11) orientam que, independentemente da categoria de classificação do estabelecimento, existem três objetivos para os quais o gerenciamento das empresas de hospitalidade deve estar orientado. Os objetivos devem garantir que: (a) o convidado se sinta bem-vindo; (b) as instalações funcionem para o convidado; e (c) a operação ofereça serviço enquanto gera lucro. Para auxiliar no alcance desses objetivos, estão incluídos nesse contexto os gerentes do setor da hospitalidade. Esses gerentes enfrentam ambientes de trabalho mais incertos e complexos do que outros setores devido às diferenças culturais, de climas e de ambientes de negócios em relação a seus valores pessoais.

Os gerentes de hospitalidade têm metas, implícitas e explícitas, e responsabilidades para assegurar a sobrevivência e o sucesso contínuo de suas organizações. Além disso, os gerentes também têm seus próprios interesses pessoais, como a progressão e o desenvolvimento profissional da sua carreira. Para cumprir as metas estabelecidas, os gerentes de empresas de hospitalidade assumem atividades do papel gerencial padrão, tais como planejar, organizar, comandar, coordenar, motivar e controlar. Essas atividades também estão incorporadas nas funções dos gerentes de alimentos e bebidas (DAVIS et al., 2012, p.11; BARROWS, 2008, p.421).

O processo de planejamento consiste em definir políticas básicas que envolvem várias áreas, tais como finanças, marketing e operações. A área financeira é responsável pela previsão de lucros e restrição de custos dos alimentos, bebidas e mão de obra. A área de marketing define os mercados-alvos a serem atendidos, promove a diferenciação do estabelecimento em relação aos concorrentes e transmite a imagem do negócio ao mercado na tentativa de atrair e reter os clientes. $\mathrm{Na}$ área de operações são definidos os principais objetivos da operação das instalações de alimentos e bebidas, bem como os métodos a serem utilizados para alcançar esses objetivos (DAVIS et al., 2012, p.15).

O processo de organização atribui a cada um dos colaboradores as tarefas necessárias para cumprir os objetivos da empresa. Para isso, os colaboradores precisam receber a descrição do trabalho que detalha a proposta das suas tarefas e as responsabilidades inerentes a elas. Nesse momento, o 
gerente de alimentos e bebidas tem papel importante na organização, auxiliando no detalhamento das atividades e na identificação de necessidade de treinamento para o pessoal. A motivação do pessoal do departamento de alimentos e bebidas pode ser conseguida quando o gerente oferece ajuda aos seus subordinados, gerando, assim, um espírito de equipe no grupo. Outra forma de motivar é oferecer treinamento aos colaboradores iniciantes, diminuindo a ansiedade e insegurança dessas pessoas quando estas assumirem novas responsabilidades. Por fim, a atividade de controle visa comparar o desempenho atual dos colaboradores com o que foi planejado, tomando ações corretivas para os grandes desvios e traçando novos procedimentos para evitar os mesmos erros no futuro (DAVIS et al., 2012, p.15; BARROWS, 2008, p.431-434).

As ações dos gerentes de alimentos e bebidas nem sempre são consistentes, pois são realizadas em função do inesperado, resultante de um ambiente que está em constante mudança. Dessa forma, o trabalho do gerente é caracterizado pela variabilidade, variedade e volatilidade. Contudo, cada uma dessas atividades está atrelada a uma função de gerenciamento e, como cada um dos gerentes, inclusive os do setor da hospitalidade, é corresponsável pelo sucesso da sua organização, deve assumir as obrigações dessas funções. Em geral, o desempenho dos gerentes é medido pelo grau de sucesso no alcance das metas estipuladas e a efetividade do seu desempenho é sustentada pelas suas competências, tais como conhecimento e habilidades. No setor de alimentos e bebidas não é diferente, ou seja, os gerentes precisam de conhecimento suficiente para conduzir os negócios estrategicamente. Devem ser habilidosos para se relacionar bem com os funcionários e clientes, cumprir as metas operacionais dentro das limitações financeiras e atender rapidamente às solicitações dos clientes para manter ou elevar o nível de qualidade de serviço no atendimento. Dentro de um contexto internacional de trabalho o geren- te precisa, ainda, ter competência para poder lidar adequadamente com as diversidades culturais. Em grandes corporações o gerente de alimentos e bebidas é, comumente, um profissional contratado para gerenciar um grupo de pessoas que estão distribuídas em vários níveis subordinados a ele. No caso de pequenos estabelecimentos, o próprio proprietário, geralmente, desempenha o papel do gerente de alimentos e bebidas (DAVIS et al., 2012, p.12).

Para Davis et al. (2012, p.18), dentre os inúmeros objetivos e responsabilidades de um departamento de alimentos e bebidas, existem três que se destacam devido à importância: (1) fornecer produtos e serviços para mercados definidos, buscando atender ou superar as expectativas geradas pelos clientes; (2) comprar, receber, armazenar, manusear e preparar os alimentos e bebidas dentro do estabelecimento para fornecer ao consumidor final; e (3) criar um sistema eficiente de controle para: monitorar preços; compilar informações de custo e venda para elaborar previsões de margem; comparar custos e vendas realizados com os planejados e efetuar ações corretivas para os grandes desvios; treinar, dirigir e monitorar os colaboradores; cooperar com outros departamentos; além de criar um instrumento para obter as avaliações dos clientes sobre os produtos e serviços, e utilizá-las como feedback na elaboração de planos de melhoria para o negócio.

O gerenciamento do departamento de alimentos e bebidas é considerado o mais técnico e complexo devido às fortes pressões, externas e internas, exercidas sobre ele. Pressões externas, como políticas governamentais, variações da economia, mudanças sociais e desenvolvimento tecnológico são forças originadas fora da empresa e as ações internas têm pouco efeito para resolver essas questões. Já as pressões internas, como perecibilidade e desperdício de alimentos, furtos nas cozinhas, absenteísmo, treinamento de pessoal e controle financeiro, são problemas gerados dentro da organização e durante a ope- 
ração cotidiana. Nesse caso, as ações internas podem resolver essas questões devido à possibilidade de identificação e eliminação da causa raiz do problema. Gerenciar alimentos e bebidas é uma atividade mais complexa do que gerenciar quartos de hotéis, pois isso exige um conjunto de conhecimentos e grau de criatividade maior do que o necessário para o gerenciamento de acomodações (DAVIS et al., 2012, p.19; RILEY, 2005).

A Gestão em Alimentos e Bebidas (Food and Beverage Management) é vista como um dos pilares centrais que definem a Gestão da Hospitalidade, e tem seu foco subdividido em Produção de Alimentos e Serviços de Alimentação. É considerada, ainda, uma das disciplinas mais importantes na maioria dos cursos de Hospitalidade ao redor do mundo. Os estudos nesse campo de pesquisa já se mostram consolidados no exterior, uma vez que o setor de serviço em alimentos e bebidas se tornou altamente lucrativo. Somente na América do Norte existe mais de 1 milhão de operações de alimentos e bebidas que faturam algo próximo de meio trilhão de dólares. Todos os dias, as pessoas ao redor do mundo compram alimentos enquanto trabalham, viajam ou desfrutam do seu tempo de lazer. Quando o padrão de vida das pessoas melhora, elas ficam propensas a gastar mais do seu dinheiro em atividades de lazer, incluindo comer fora do lar. Nos Estados Unidos, por exemplo, um americano gasta quase metade do valor despendido em alimentação (em torno de $40 \%$ ) com refeições realizadas fora de casa. Isso corrobora o fato de os Estados Unidos serem o país que gera a maior receita em serviços de alimentação no mundo (TEWS \& HOOF, 2011; BARROWS, 2008, p.422; WOOD, 2007).

Barrows (2008, p.435) discorre sobre a situação do setor de alimentos e bebidas nos países com maior destaque nessa área. Segundo o autor, os Estados Unidos e a China dominam o mercado de serviço de alimentação em termos de quantidade de estabelecimentos e valor de vendas em geral. O país norte-americano se destaca devido ao elevado valor gasto pela população com serviços de alimentação, que é abastecido por grandes redes multinacionais, além do grande número de conceitos e tendências gerados lá. A China ganhou notoriedade devido à sua cultura alimentar e ao grande número de estabelecimentos de propriedade independente existentes no país. Apesar de o Japão ser o segundo país com maior participação em valor de vendas na indústria da alimentação, apresenta um crescimento lento. Os serviços que mais crescem são os de entrega (delivery), comida para levar (takeaway) e cafeterias (coffee shops). No Brasil, a indústria de alimentos e bebidas foi afetada pelas recentes crises econômicas e, também, pelo fato de muitos brasileiros não terem condições financeiras para comer fora de casa com frequência. Um dos maiores conceitos de restaurante no Brasil é a alimentação por quilo, onde as pessoas pagam suas refeições de acordo com o peso da comida que é servida pelo próprio consumidor em seu prato. O Reino Unido tem a maior diversidade de serviço de alimentação e maior número de estabelecimentos afiliados em cadeias de fornecimento dos que os outros países mencionados nesta seção. Os consumidores dessa região estão migrando seu consumo para pubs que oferecem opções de menu de luxo. Já os russos gastam em torno de $15 \%$ do valor despendido em alimentação para comer fora de casa, pois ainda consideram esse costume uma prática de luxo. As barracas e quiosques de rua dominam o mercado e representam $40 \%$ dos estabelecimentos, mas apenas $4 \%$ das unidades de alimentação estão afiliadas em cadeias de fornecimento. Por fim, a Alemanha foi o país onde o setor de alimentação enfrentou as maiores dificuldades, devido a crises econômicas, inflação e desemprego. Contudo, ainda apresenta uma alta proporção de restaurantes que oferecem serviço completo.

Os mercados dos países descritos acima indicam várias diferenças no setor de alimentos e bebidas ao redor do mundo. 
Essas características mostram, também, que esse setor está passando por mudanças que decorrem de questões demográficas, do aumento de renda das famílias e do maior tempo despendido pelas pessoas com lazer. $\mathrm{O}$ aumento de viagens para outros países traz uma demanda maior de internacionalização da cozinha para os estabelecimentos de alimentação. Cada vez mais as pessoas procuram sabores e experiências diferentes quando buscam a alimentação fora de casa. Na mesma tendência, a demanda por comida saudável também tem aumentado e, devido a isso, esse tipo de alimento está sendo incluído nos menus de todos os tipos de restaurante. Além da comida saudável, os estabelecimentos passaram a promover produtos subutilizados, como algumas espécies de frutos do mar, por exemplo, para mitigar os riscos de incerteza no fornecimento e diminuir os custos de forma geral (BARROWS, 2008, p.437). Outra decisão adotada pelas empresas para diminuir os custos foi a de abrirem seus estabelecimentos em imóveis mais simples e em locais não tão privilegiados (BARROWS, 2008, p.440).

$\mathrm{O}$ setor de alimentos e bebidas tem sofrido com algumas ineficiências operacionais. Devido ao fato de o trabalho nessa área ser intenso, a sua eficiência está diretamente ligada ao nível de gerenciamento de recursos humanos. O gerenciamento de recursos humanos ainda é um desafio para os gerentes desse setor, devido à escassez de trabalho em muitas regiões e, também, por não apresentar uma opção de carreira viável às pessoas. As empresas desse segmento precisam aprimorar várias áreas do gerenciamento de recursos humanos (recrutamento, seleção, compensação, treinamento e desenvolvimento) para poder disputar funcionários com outras empresas, inclusive de outros setores. Assim, para diminuir a rotatividade de pessoal os estabelecimentos de alimentos e bebidas precisam oferecer melhores condições de trabalho e progressão de carreira mais atrativa. Em face de todas essas variáveis, os gerentes de alimentos e bebidas precisam estar preparados para se antecipar às mudanças do ambiente externo, bem como gerenciar suas operações estrategicamente (BARROWS, 2008, p.440).

\section{PESQUISA BIBLIOGRÁFICA}

A pesquisa científica auxilia na compreensão de um determinado assunto de uma forma melhor do que qualquer outro tipo de trabalho. Por meio da pesquisa e da publicação da mesma é possível solucionar um enigma ou descobrir algo novo e desconhecido, contribuindo, assim, para o enriquecimento do conhecimento humano. Sobre isso, muitos estudantes, ou mesmo pesquisadores em nível inicial, confundem a pesquisa bibliográfica com revisão bibliográfica ou revisão de literatura (BOOTH, COLOMB \& WILLIAMS, 2005; LIMA \& MIOTO, 2007).

A revisão de literatura, também chamada de revisão bibliográfica, é um requisito básico para elaboração da pesquisa científica. A revisão da literatura é a etapa inicial no desenvolvimento do conhecimento científico, pois tende a possibilitar e contextualizar a identificação de lacunas que criam oportunidades para fomentar novas pesquisas. As revisões de literatura são o resultado da análise e síntese dos conteúdos disponibilizados por outros estudos científicos já publicados sobre um determinado tema. Elas precisam ser sistemáticas, didáticas, atuais no contexto do tema revisado e, preferencialmente, quando possível, abranger os clássicos da área ou do tema em questão, pois possuem um papel importante na transferência da informação entre os pesquisadores e suas comunidades científicas, bem como para seus leitores não especialistas (BOTELHO, CUNHA \& MACEDO, 2011; LIMA \& MIOTO, 2007; FIGUEIREDO, 1990).

Outro conceito que também pode ser confundido com a pesquisa bibliográfica é a modalidade de estudo chamada de pesquisa documental. Esse método visa obter informações de documentos que não são considerados científicos, tais como 
matérias de jornais e revistas, cartas, filmes, fotos, pôsteres e outros tipos de apresentações. A natureza das fontes é o principal diferencial entre a pesquisa documental e a pesquisa bibliográfica. A pesquisa documental tem seu processo de investigação vinculado a fontes primárias, que são dados originais gerados pelos fatos da realidade empírica e que precisarão ser analisados pelo pesquisador (SÁ-SILVA, ALMEIDA \& GUINDANI, 2009).

Por outro lado, a pesquisa bibliográfica está atrelada a fontes secundárias, ou seja, informações que já foram analisadas, estudadas, trabalhadas e divulgadas às comunidades científicas por outros pesquisadores. Nesse sentido, pesquisa bibliográfica é um método composto por um conjunto de procedimentos que são aplicados, exclusivamente, sobre fontes bibliográficas, como livros acadêmicos, handbooks, artigos científicos, dissertações, teses, entre outras fontes científicas, na busca da solução de um problema, de hipóteses ou proposições. Eventualmente, pode-se, também, recorrer às bases de dados de pesquisas acadêmicas como, por exemplo, EBSCO, ProQuest ou Google Acadêmico como instrumento de auxílio para o levantamento de dados da pesquisa. O objetivo da pesquisa bibliográfica é avaliar as tendências (de forma longitudinal) sobre um determinado assunto, teoria, disciplina ou campo de estudo. Também identifica as principais teorias, objetos de estudo, elementos, categorias, dimensões, estudiosos e instituições mapeando a estrutura científica de uma teoria, disciplina ou área de estudo. Dessa forma, a pesquisa bibliográfica pode oferecer subsídios e diferentes perspectivas que aumentam o conhecimento e oferecem avanços e descobertas acerca do tema investigado (MOTA et al., 2014; PIZZANI, SILVA, BELLO \& HAYASHI, 2012; SÁSILVA, ALMEIDA \& GUINDANI, 2009; LIMA \& MIOTO, 2007; BOCCATO, 2006).

Boccato (2006) enfatiza que é extremamente importante que o pesquisador planeje o seu processo de pesquisa biblio- gráfica. Esse planejamento deve considerar todas as atividades da pesquisa, como a definição do tema, a construção do trabalho e a forma de divulgação dos resultados. Para tal, Lima e Mioto (2007) sugerem uma sequência de procedimentos para a construção de uma pesquisa bibliográfica que contempla quatro fases. A primeira fase, chamada de Elaboração do projeto de pesquisa, consiste na escolha do tema, na elaboração do problema de pesquisa e no plano de como as respostas para as perguntas desse problema serão obtidas. Nessa fase, o tema deve ser cientificamente útil para o desenvolvimento da área na qual a pesquisa está sendo realizada. Ele precisa, também, atender a outros interesses, além dos do próprio pesquisador, e auxiliar a comunidade científica no desenvolvimento de novos estudos. Sugere-se, ainda, a identificação de termos e palavras-chave que expressem o tema, não só em Português, mas também em outras línguas nas quais o pesquisador julgue necessário para a busca dos documentos científicos. A segunda fase é a Investigação das soluções, na qual acontece, em um determinado intervalo de tempo, o levantamento da bibliografia e o estudo dos dados e informações por meio dos resumos e alguns detalhes oferecidos pela própria base de dados. Nessa etapa o pesquisador deve buscar, atentamente, toda a informação relacionada ao tema investigado que esteja disponível na base de dados escolhida. Atualmente, existem várias bases de dados de produção científica que podem ser acessadas eletronicamente. Algumas delas oferecem acesso livre e gratuito, enquanto outras disponibilizam seus conteúdos somente mediante a senha, que é obtida após a aquisição ou assinatura do serviço. Na terceira fase, conhecida como Análise explicativa das soluções, é feita a análise e o exame do conteúdo das publicações selecionadas. É nela que a capacidade crítica e analítica do pesquisador determina e justifica os dados que serão mantidos no material selecionado, podendo classificá-los, ainda, em categoriais conceituais. A quarta e última fase, Síntese inte- 
gradora, é o resultado final do trabalho de investigação, análise e reflexão dos documentos. Esse é o momento de imersão do pesquisador no material de estudo obtido, para ler, anotar e questionar, com o objetivo de refletir sobre as proposições de soluções. Essas reflexões são apoiadas pelas publicações selecionadas para o estudo e apresentadas nos resultados da pesquisa, em conjunto com a revisão de literatura, que sintetiza o tema abordado por meio de estudos anteriores (PIZZANI et al., 2012; BOCCATO, 2006).

Para Treinta, Filho, Sant'anna e Rabelo (2014), os pesquisadores devem sempre se perguntar se todos os artigos relacionados ao tema pesquisado foram encontrados e selecionados. Isso faz da pesquisa bibliográfica um processo contínuo, no qual o pesquisador precisa ficar atento a novas produções científicas que possam encorpar a amostra do estudo. Ainda que não seja foco deste estudo, existe, também, a possibilidade de estudos bibliométricos atrelados à pesquisa bibliográfica. A bibliometria é uma técnica quantitativa utilizada para tratar grandes volumes de informação, em que uma análise de conteúdo bibliográfico usual não seria viável. Em outras palavras, a técnica utiliza, em geral, análises de citações e cocitações, por meio de métodos estatísticos e matemáticos, buscando identificar as principais teorias, os autores e/ou pesquisadores mais produtivos. Essa técnica possibilita um mapeamento da estrutura intelectual, por meio de uma rede de relações, de uma teoria, disciplina ou área (MARIETTO \& MACCARI, 2015; SERRA, FERREIRA, PEREIRA \& LISSONI, 2008).

\section{MÉTODO}

Para a realização deste estudo foram efetuadas diversas pesquisas nas bases de dados EBSCO e Food and Beverage Management Commons, sendo que essa segunda base de dados foi acessada através da Digital Commons Network. As palavras-chave utilizadas para a procura dos artigos foram: "Gestão em Alimentos e Bebidas", "Gestão de Alimentos e Bebidas", "Food and Beverage Management", "Gestión em Alimentos y Bebidas" e "Alimentos y Bebidas Gestión", todas elas digitadas entre aspas. Na base de dados EBS$C O$ foi aplicado um filtro na busca que limitou a exibição de estudos publicados somente em revistas acadêmicas. Na base de dados Food and Beverage Management Commons não foi utilizado nenhum tipo de filtro adicional além das palavras-chaves digitadas entre aspas. As buscas resultaram em uma amostra total de 1321 artigos, sendo 459 da EBSCO e 862 da Food and Beverage Management Commons.

Todos os resultados (hyperlinks) das buscas, exibidos nas páginas dos bancos de dados, foram copiados e colados numa planilha do Microsoft Excel. Esse conteúdo foi organizado de forma que cada título de artigo fosse alocado em células individuais na planilha, possibilitando, assim, trabalhar com os dados em formato de tabela. Esses títulos foram, então, classificados em ordem alfabética e formatados na planilha para que pudessem receber as informações complementares de identificação dos artigos (Ano, Área da Administração e Objeto de estudo) na mesma linha do título do documento.

Assim que os dados foram organizados, a primeira tarefa foi analisar toda a lista de artigos e retirar os títulos repetidos. Para isso, foi utilizado o auxílio da fórmula "SE" do Microsoft Excel, que identificou os artigos com títulos iguais. Esses títulos foram, então, selecionados e excluídos da lista. Após essa exclusão, a lista apresentou um total de 1138 documentos para serem analisados.

Em seguida, na etapa qualitativa, cada um dos artigos da lista foi acessado para, então, serem feitas as análises dos artigos baseadas no título, ano de publicação, resumo ou abstract, palavras-chave e, quando necessário, introdução e método das pesquisas. Os estudos correlatos com o tema pesquisado tiveram seus dados complementares preenchidos na planilha (Ano, 
Área da Administração e Objeto de estudo). Os artigos que não apresentaram nenhuma relação com o tema da pesquisa foram excluídos da lista. Depois de finalizada a análise de todos os textos e fichamento dos artigos selecionados para a pesquisa, a quantidade da amostra diminuiu para 375 artigos. Como o ano corrente (2016) ainda não havia sido finalizado na ocasião da realização deste estudo, foram retirados da lista, também, 7 (sete) artigos publicados nesse ano. Por fim, obtivemos uma moldura amostral final de 368 artigos que apresentaram alguma relação com as áreas de gestão em alimentos e bebidas.

A partir da lista com a amostragem final (368 artigos), foram geradas novas planilhas no Microsoft Excel com tabelas e gráficos dinâmicos, que são recursos oferecidos pelo próprio software, os quais exibiram as quantidades e percentuais de publicações classificadas por década, área da administração e objeto de estudo. Por meio dessas tabelas e gráficos foram obtidos os valores descritos na seção Resultados, que poderão servir de indicadores para nortear a realização de novas pesquisas nas áreas de gestão em alimentos e bebidas. $\mathrm{O}$ fluxograma a seguir oferece uma visão geral das etapas do método, desde a busca nas bases de dados até a seleção da amostragem final.

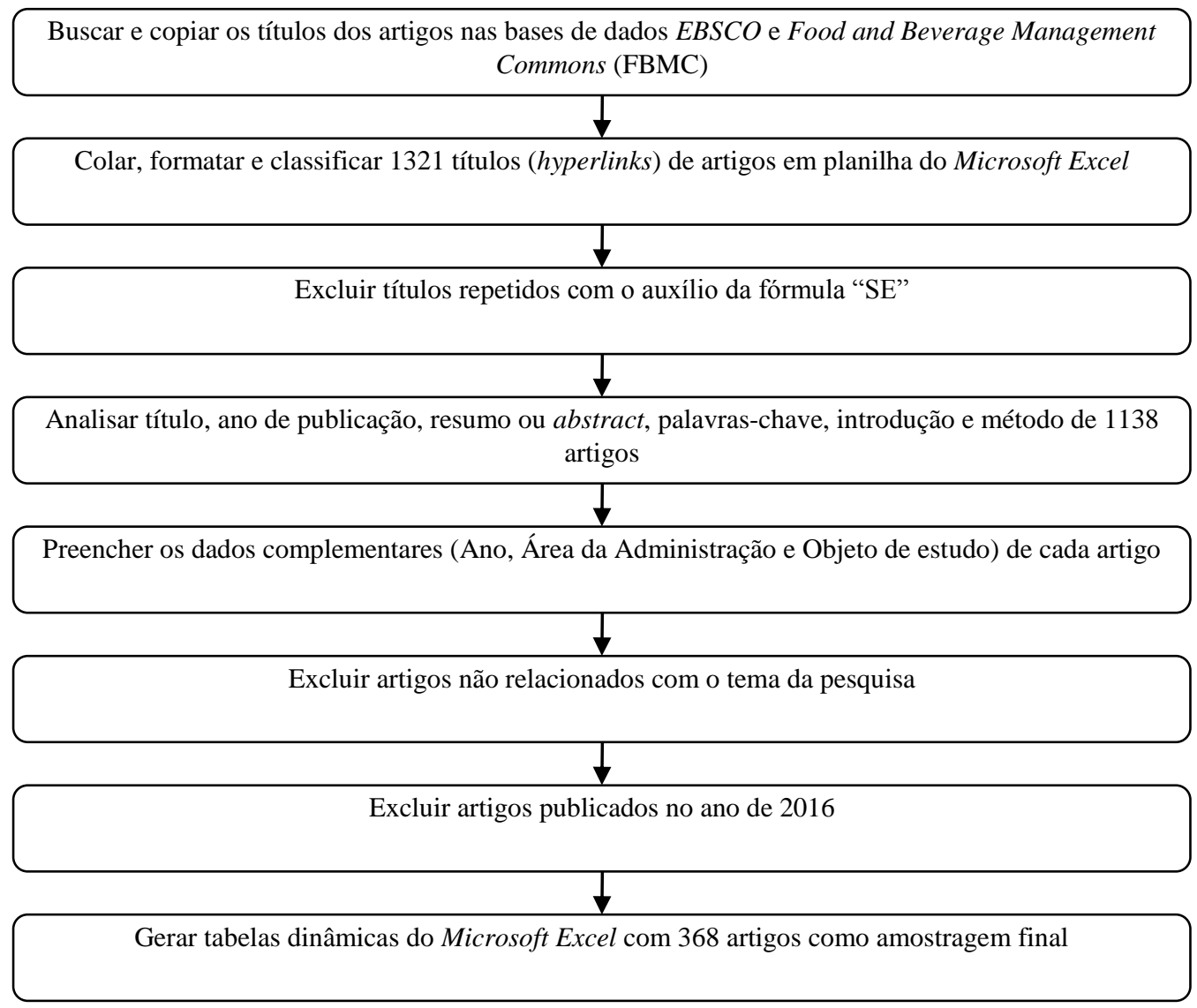

Figura 1 Fluxograma do procedimento de seleção dos artigos

\section{RESULTADOS DA PESQUISA}

Os primeiros artigos a serem publicados são da década de 1960, em que a mais antiga data do ano de 1962 e apresen- ta um estudo sobre os salários gerenciais no setor de serviço de alimentação. Ainda nessa década foram publicados mais dois 
artigos, no ano de 1966. Um deles relacionado à área de Recursos Humanos de Hotel e o outro a questões ligadas ao Conceito do Negócio de Restaurante de Hotel. Todos os três artigos tiveram seus estudos publicados pelo Journal Cornell Hospitality Quarterly, que está associado à Cornell University School of Hotel Administration, situada na cidade de Ithaca, estado de New York, nos Estados Unidos da América.

A década de 1970 contabiliza um total de 07 artigos, publicados entre os anos de 1976 e 1979. O único artigo de
1976 estudou a Gestão de Preços em Hotel. No ano de 1977 encontramos, também, apenas um artigo, que está relacionado a Recursos Humanos de Restaurante. $\mathrm{O}$ ano com mais publicações nessa década é o de 1978, com três artigos. Dois deles na área de Recursos Humanos de Hotel e Setor de Serviço de Alimentação, respectivamente, e um em Gestão de Custos de Restaurante. No final desse período, o ano de 1979 teve duas publicações, sendo um artigo na área de Recursos Humanos e o outro em Gestão de Custos, ambos em Hotel.

\begin{tabular}{|c|c|c|c|c|}
\hline & & Dé & das & \\
\hline Área & Objeto & 1960 & 1970 & Total \\
\hline \multirow{3}{*}{ Recursos Humanos } & Hotel & 1 & 2 & 3 \\
\hline & Setor de Serviço de Alimentos & 1 & 1 & 2 \\
\hline & Restaurante & - & 1 & 1 \\
\hline Gestão de Preços & Hotel & - & 1 & 1 \\
\hline \multirow{2}{*}{ Gestão de Custos } & Hotel & - & 1 & 1 \\
\hline & Restaurante & - & 1 & 1 \\
\hline Conceito do Negócio & Restaurante de Hotel & 1 & - & 1 \\
\hline Total Geral & & 3 & 7 & 10 \\
\hline
\end{tabular}

Quadro 1 Quantidade de Áreas da Administração e de Objetos investigados nas publicações das décadas de 1960 e 1970

O número total de publicações na década de 1980 chegou a 31 artigos, ou seja, um número três vezes maior que o total das duas décadas anteriores juntas. $\mathrm{O}$ único ano que não apresenta artigo publicado nesse período é o de 1982. A área com mais estudos publicados é a de Recursos Humanos com 12 textos, seguida das áreas de Conceito de Negócios com 09 e Gestão da Marca com 04. As áreas de Atendimento e ambiente e Gestão de Custos contabilizaram 02 artigos cada uma. E, por fim, as áreas de Controles e Gestão de Preços com uma publicação cada. Os objetos de pesquisa mais estudados foram Hospitalidade (19 artigos), Hotel (05 artigos), Restaurante (03 artigos), Clube e Hotel e restaurante (02 artigos cada).
$\mathrm{O}$ período seguinte apresentou publicações em todos os anos da década de 1990, com destaque para o ano de 1995 com 07 estudos publicados. A quantidade total de produções praticamente se manteve em relação à década anterior, com 37 artigos. Novamente, a área de Recursos Humanos foi a mais estudada, com 09 trabalhos apresentados. As áreas de Controles e Conceito do Negócio aparecem a seguir com 06 artigos cada uma. Na sequência estão as áreas de Atendimento e ambiente (05 artigos), Gestão de Preços (04 artigos), Tecnologia da Informação (03 artigos) e Higiene e Segurança (02 artigos). As áreas de Gestão Financeira e Gestão de Custos fecham o período com uma publicação cada. 
Gestão em Alimentos e Bebidas: Indicadores para um Novo Campo de Estudos no Brasil

\begin{tabular}{|c|c|c|c|}
\cline { 2 - 3 } \multicolumn{1}{c|}{} & \multicolumn{2}{c}{ Décadas } & \multicolumn{2}{c}{ Total por Área } \\
\hline Área & $\mathbf{1 9 8 0}$ & $\mathbf{1 9 9 0}$ & 21 \\
\hline Recursos Humanos & 12 & 9 & 15 \\
\hline Conceito do Negócio & 9 & 6 & 7 \\
\hline Atendimento e ambiente & 2 & 5 & 7 \\
\hline Controles & 1 & 6 & 5 \\
\hline Gestão de Preços & 1 & 4 & 4 \\
\hline Gestão da Marca & 4 & - & 3 \\
\hline Gestão de Custos & 2 & 1 & 3 \\
\hline Tecnologia da Informação & - & 3 & 2 \\
\hline Higiene e segurança & - & 2 & $\mathbf{6 8}$ \\
\hline Gestão Financeira & - & 1 & $\mathbf{3}$ \\
\hline Total Geral & $\mathbf{3 1}$ & $\mathbf{3 7}$ & \multicolumn{2}{|c|}{} \\
\hline
\end{tabular}

Quadro 2 Quantidade de Áreas da Administração nas publicações das décadas de 1980 e 1990

Somente três objetos foram alvos de estudos na década de 1990: Hospitalidade, Restaurante e Hotel. O objeto escolhido em 18 estudos foi Hospitalidade, enquanto Restaurante figurou em 11 publicações e Hotel em 08 textos. Alguns trabalhos selecionaram, especificamente, hotel e restaurante de forma conjunta em seus estudos, diferentemente de outros artigos que consideraram unicamente cada um desses objetos em suas pesquisas. Dessa forma, consideramos o objeto Hotel e restaurante (conjugados) um objeto diferente de Hotel e Restaurante estudados isoladamente.

\begin{tabular}{|c|c|c|c|}
\cline { 2 - 3 } \multicolumn{1}{c|}{} & \multicolumn{2}{c|}{ Décadas } & Total por Objeto \\
\hline Objeto & $\mathbf{1 9 8 0}$ & $\mathbf{1 9 9 0}$ & 37 \\
\hline Hospitalidade & 19 & 18 & 14 \\
\hline Restaurante & 3 & 11 & 13 \\
\hline Hotel & 5 & 8 & 2 \\
\hline Clube & 2 & - & 2 \\
\hline Hotel e restaurante & 2 & - & $\mathbf{6 8}$ \\
\hline Total Geral & $\mathbf{3 1}$ & $\mathbf{3 7}$ & \\
\hline \multicolumn{2}{|c|}{} \\
\hline
\end{tabular}

O total de publicações entre os anos de 2000 e 2009 mais que dobrou em relação à década anterior, chegando a 91 artigos aceitos pelos periódicos acadêmicos. $\mathrm{O}$ ano com mais estudos divulgados foi o de 2009, embora encontremos pesquisas publicadas em todos os anos desse decênio. Assim como na década de 1990, questões referentes à área de Recursos Humanos foram as mais discutidas, com 35 artigos, apresentando quase duas vezes mais trabalhos publicados do que a segunda área mais estudada, Atendimento e Ambiente, com 18 textos. A terceira área mais abordada foi a de Conceito de Negócio com 16 artigos, seguida de Gestão de Custos (05 artigos), Higiene e segurança e Gestão de Preços (03 artigos cada) e Produção e operações, Cardápio e Cadeia de Suprimentos (02 artigos cada). As áreas de Gestão da Marca, Qualidade, Tecnologia da Informação, Gestão Financeira e Negócios Internacionais apresentaram, ainda, 01 publicação cada. 
Gestão em Alimentos e Bebidas: Indicadores para um Novo Campo de Estudos no Brasil

\begin{tabular}{|c|c|}
\hline Área & Década de 2000 \\
\hline Recursos Humanos & 35 \\
\hline Atendimento e ambiente & 18 \\
\hline Conceito do Negócio & 16 \\
\hline Gestão de Custos & 5 \\
\hline Higiene e segurança & 3 \\
\hline Gestão de Preços & 3 \\
\hline Produção e Operações & 2 \\
\hline Cardápio & 2 \\
\hline Cadeia de Suprimentos & 2 \\
\hline Gestão da Marca & 1 \\
\hline Qualidade & 1 \\
\hline Tecnologia da Informação & 1 \\
\hline Gestão Financeira & 1 \\
\hline Negócios Internacionais & 1 \\
\hline Total Geral & 91 \\
\hline
\end{tabular}

Quadro 4 Quantidade de Áreas da Administração nas publicações da década de 2000

O objeto mais evidente nos trabalhos da década de 2000 foi o Restaurante, protagonizado em 39 artigos publicados. O segundo mais estudado foi Hotel, figurando em 23 pesquisas, seguido por Hospitalidade com 17 artigos. Alguns novos objetos de estudo surgiram nesse período, dentre eles, Resort, Catering e Clube, que foram escolhidos em 02 estudos no período. Outros, como Cafeteria de Faculdade, Vinícola, Restaurante Fast Food, Setor de Serviço de Alimentos, Comida de rua e Hotel e restaurante, foram alvos de estudo de 01 artigo cada um.

\begin{tabular}{|c|c|}
\hline Objeto & Década de 2000 \\
\hline Restaurante & 39 \\
\hline Hotel & 23 \\
\hline Hospitalidade & 17 \\
\hline Resort & 2 \\
\hline Catering & 2 \\
\hline Clube & 2 \\
\hline Cafeteria de Faculdade & 1 \\
\hline Vinícola & 1 \\
\hline Restaurante Fast Food & 1 \\
\hline Setor de Serviço de Alimentos & 1 \\
\hline Comida de rua & 1 \\
\hline Hotel e restaurante & 1 \\
\hline Total Geral & $\mathbf{9 1}$ \\
\hline
\end{tabular}

Quadro 5 Quantidade de Objetos investigados nas publicações da década de 2000

Na década atual, de 2010 até 2015, foram encontradas 199 publicações relacionadas à Gestão em Alimentos e Bebidas. Além da já evidenciada área de Recursos
Humanos, escolhida em 53 artigos, a área de Atendimento e Ambiente também passou a figurar na maioria das pesquisas em questão, estudada em 48 publicações. A 
área de Conceito do Negócio participou de 34 trabalhos. Em seguida, uma nova área, Consciência Ambiental, aparece em 15 estudos realizados. A área de Cadeia de Suprimentos foi foco de investigação em 08 artigos, enquanto Gestão da Marca foi escolhida em 07 pesquisas. Gestão de Preços e Controles foram escolhidas em 06 pesquisas cada e, fechando essa década, aparecem as áreas de Gestão de Custos (05 artigos), Cardápios e Produção e operações (04 artigos cada), Tecnologia da Informação, Gestão Financeira e Higiene e segurança (02 artigos cada) e Negócios Internacionais, Marketing e Gestão de Estoques com 01 estudo cada.

\begin{tabular}{|c|c|}
\hline Área & Década de 2010 \\
\hline Recursos Humanos & 53 \\
\hline Atendimento e ambiente & 48 \\
\hline Conceito do Negócio & 34 \\
\hline Consciência Ambiental & 15 \\
\hline Cadeia de Suprimentos & 8 \\
\hline Gestão da Marca & 7 \\
\hline Gestão de Preços & 6 \\
\hline Controles & 6 \\
\hline Gestão de Custos & 5 \\
\hline Cardápio & 4 \\
\hline Produção e Operações & 4 \\
\hline Tecnologia da Informação & 2 \\
\hline Gestão Financeira & 2 \\
\hline Higiene e segurança & 2 \\
\hline Negócios Internacionais & 1 \\
\hline Marketing & 1 \\
\hline Gestão de Estoques & 1 \\
\hline Total Geral & $\mathbf{1 9 9}$ \\
\hline Quadro 6 Quantidade de Áreas da Administração nas publicações da década de 2010 & \\
\hline
\end{tabular}

Novamente o Restaurante foi o objeto mais presente nos estudos desse período, protagonizado em 85 pesquisas, seguido por Hospitalidade e Hotel com, respectivamente, 54 e 38 artigos. Setor de Serviço de Alimentos foi estudado em 04 pesquisas, enquanto Hotel e pousada e Vinícola foram selecionados em 03 trabalhos cada um. Restaurante de Hotel apareceu em 02 pesquisas, ao passo que Catering, Pousadas, Gastronomia, Agência de viagem e hotel, Restaurante Fast Food, Bar, Festival, Hotel e restaurante, Hotel Cassino e Hotel e catering figuraram em apenas 01 artigo cada um. Da mesma forma que Hotel e restaurante, mencionado anteriormente, Hotel e pousada, Agência de viagem e hotel e Hotel e catering foram considerados objetos diferentes por terem sidos estudados de forma conjunta nos artigos selecionados.

\begin{tabular}{|c|c|}
\hline Objeto & Década de 2010 \\
\hline Restaurante & 85 \\
\hline Hospitalidade & 54 \\
\hline Hotel & 38 \\
\hline
\end{tabular}


Gestão em Alimentos e Bebidas: Indicadores para um Novo Campo de Estudos no Brasil

\begin{tabular}{|c|c|}
\hline Setor de Serviço de Alimentos & 4 \\
\hline Hotel e pousada & 3 \\
\hline Vinícola & 3 \\
\hline Restaurante de Hotel & 2 \\
\hline Catering & 1 \\
\hline Pousadas & 1 \\
\hline Gastronomia & 1 \\
\hline Agência de Viagem e hotel & 1 \\
\hline Restaurante Fast Food & 1 \\
\hline Bar & 1 \\
\hline Festival & 1 \\
\hline Hotel e restaurante & 1 \\
\hline Hotel Cassino & 1 \\
\hline Hotel e catering & 1 \\
\hline Total Geral & $\mathbf{1 9 9}$ \\
\hline
\end{tabular}

Quadro 7 Quantidade de Objetos investigados nas publicações da década de 2010

No período total analisado, desde a primeira publicação encontrada, em 1962, até o ano de 2015, podemos notar um aumento exponencial na quantidade de produção científica relacionada à Gestão em Alimentos e Bebidas. Destaque para as décadas de 1980, 2000 e 2010, que apre- sentam em seus anos um número total de publicações maior do que as décadas anteriores juntas. Em termos percentuais, a década de 2000 representa uma quantidade de artigos equivalente a $54 \%$ de todo o estudo realizado.

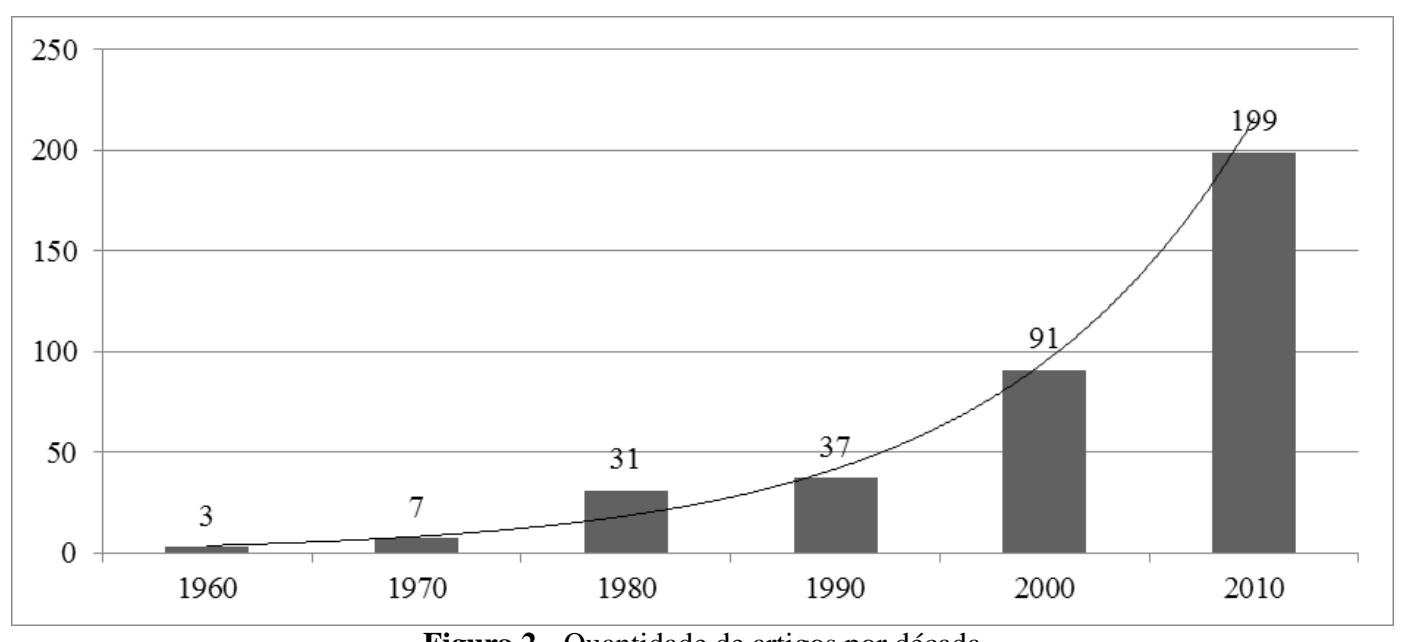

Figura 2 - Quantidade de artigos por década

\begin{tabular}{|c|c|c|}
\hline Década & Quantidade de artigos & \% em relação ao total de artigos \\
\hline 1960 & 3 & $1 \%$ \\
\hline 1970 & 7 & $2 \%$ \\
\hline 1980 & 31 & $8 \%$ \\
\hline 1990 & 37 & $10 \%$ \\
\hline 2000 & 91 & $25 \%$ \\
\hline 2010 & 199 & $54 \%$ \\
\hline
\end{tabular}


Gestão em Alimentos e Bebidas: Indicadores para um Novo Campo de Estudos no Brasil

\begin{tabular}{|c|c|c|}
\hline Total Geral & $\mathbf{3 6 8}$ & $\mathbf{1 0 0 \%}$ \\
\hline \multicolumn{2}{|c|}{ Quadro 8 Evolução da quantidade de publicações no período analisado }
\end{tabular}

Quadro 8 Evolução da quantidade de publicações no período analisado

Dentre todas as áreas da administração estudadas, a de Recursos Humanos é a que mais aparece nos artigos, com um total de 115 publicações. Embora a área de Atendimento e ambiente tenha aumentado sua participação nos estudos da década de 2000, ela foi selecionada em 73 textos desde o início do período analisado, seguida pela área de Conceito do Negócio com 66 pesquisas. A seguir aparecem as áreas de
Consciência Ambiental, Gestão de Custos e Gestão de Preços (15 artigos cada uma), Controles (13 artigos), Gestão da Marca (12 artigos), Cadeia de Suprimentos (10 artigos), Higiene e segurança (07 artigos) e Cardápio, Produção e operações e Tecnologia da Informação (06 artigos cada). As áreas de Gestão Financeira, Negócios Internacionais, Marketing, Qualidade e Gestão de Estoques totalizaram 09 estudos.

\begin{tabular}{|c|c|c|c|c|c|c|c|}
\hline \multirow[b]{2}{*}{ Área } & \multicolumn{6}{|c|}{ Décadas } & \multirow[b]{2}{*}{ Total } \\
\hline & 1960 & 1970 & 1980 & 1990 & 2000 & 2010 & \\
\hline Recursos Humanos & 2 & 4 & 12 & 9 & 35 & 53 & 115 \\
\hline Atendimento e ambiente & - & - & 2 & 5 & 18 & 48 & 73 \\
\hline Conceito do Negócio & 1 & - & 9 & 6 & 16 & 34 & 66 \\
\hline Consciência Ambiental & - & - & - & - & - & 15 & 15 \\
\hline Gestão de Custos & - & 2 & 2 & 1 & 5 & 5 & 15 \\
\hline Gestão de Preços & - & 1 & 1 & 4 & 3 & 6 & 15 \\
\hline Controles & - & - & 1 & 6 & - & 6 & 13 \\
\hline Gestão da Marca & - & - & 4 & - & 1 & 7 & 12 \\
\hline Cadeia de Suprimentos & - & - & - & - & 2 & 8 & 10 \\
\hline Higiene e segurança & - & - & - & 2 & 3 & 2 & 7 \\
\hline Cardápio & - & - & - & - & 2 & 4 & 6 \\
\hline Produção e Operações & - & - & - & - & 2 & 4 & 6 \\
\hline Tecnologia da Informação & - & - & - & 3 & 1 & 2 & 6 \\
\hline Gestão Financeira & - & - & - & 1 & 1 & 2 & 4 \\
\hline Negócios Internacionais & - & - & - & - & 1 & 1 & 2 \\
\hline Marketing & - & - & - & - & - & 1 & 1 \\
\hline Qualidade & - & - & - & - & 1 & - & 1 \\
\hline Gestão de Estoques & - & - & - & - & - & 1 & 1 \\
\hline Total Geral & 3 & 7 & 31 & 37 & 91 & 199 & 368 \\
\hline
\end{tabular}

Quadro 9 Evolução das Áreas da Administração nas publicações do período analisado 


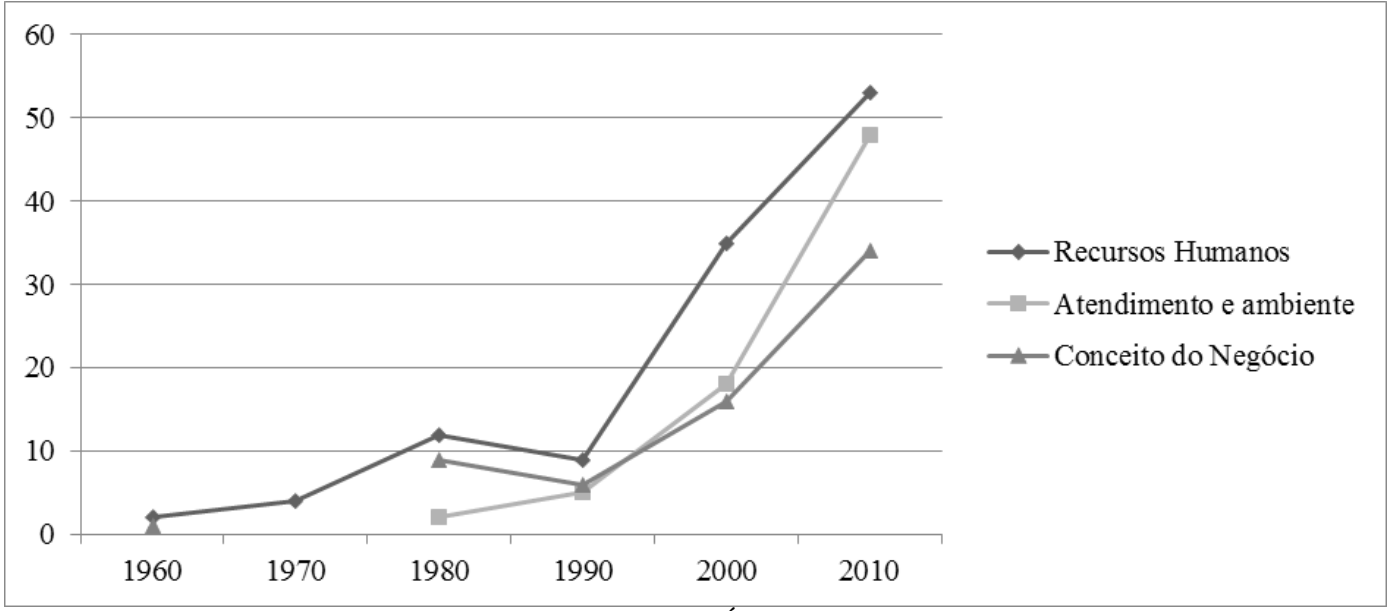

Figura 3 - Evolução das Áreas mais estudadas

Ao todo foram pesquisados $21 \mathrm{ob}-$ jetos diferentes dentro das áreas abordadas acima. O Restaurante foi o principal foco dos estudos da Gestão em Alimentos e Bebidas, sendo selecionado em 140 trabalhos publicados. Em seguida estão:
Hospitalidade com 108 artigos; Hotel com 79 textos; e Setor de Serviço de Alimentos com 07 pesquisas publicadas. Os demais objetos presentes nos trabalhos publicados totalizaram 34 estudos.

\begin{tabular}{|c|c|c|c|c|c|c|c|}
\hline \multirow[b]{2}{*}{ Objeto } & \multicolumn{6}{|c|}{ Décadas } & \multirow[b]{2}{*}{ Total } \\
\hline & 1960 & 1970 & 1980 & 1990 & 2000 & 2010 & \\
\hline Restaurante & - & 2 & 3 & 11 & 39 & 85 & 140 \\
\hline Hospitalidade & - & - & 19 & 18 & 17 & 54 & 108 \\
\hline Hotel & 1 & 4 & 5 & 8 & 23 & 38 & 79 \\
\hline Setor de Serviço de Alimentos & 1 & 1 & - & - & 1 & 4 & 7 \\
\hline Hotel e restaurante & - & - & 2 & - & 1 & 1 & 4 \\
\hline Clube & - & - & 2 & - & 2 & - & 4 \\
\hline Vinícola & - & - & - & - & 1 & 3 & 4 \\
\hline Catering & - & - & - & - & 2 & 1 & 3 \\
\hline Hotel e pousada & - & - & - & - & - & 3 & 3 \\
\hline Restaurante de Hotel & 1 & - & - & - & - & 2 & 3 \\
\hline Resort & - & - & - & - & 2 & - & 2 \\
\hline Restaurante Fast Food & - & - & - & - & 1 & 1 & 2 \\
\hline Pousadas & - & - & - & - & - & 1 & 1 \\
\hline Cafeteria de Faculdade & - & - & - & - & 1 & - & 1 \\
\hline Gastronomia & - & - & - & - & - & 1 & 1 \\
\hline Comida de rua & - & - & - & - & 1 & - & 1 \\
\hline Bar & - & - & - & - & - & 1 & 1 \\
\hline Festival & - & - & - & - & - & 1 & 1 \\
\hline
\end{tabular}


Gestão em Alimentos e Bebidas: Indicadores para um Novo Campo de Estudos no Brasil

\begin{tabular}{|l|c|c|c|c|c|c|c|}
\hline Agência de Viagem e Hotel & - & - & - & - & - & 1 & 1 \\
\hline Hotel e catering & - & - & - & - & - & 1 & 1 \\
\hline Hotel Cassino & - & - & - & - & - & 1 & 1 \\
\hline Total Geral & $\mathbf{3}$ & $\mathbf{7}$ & $\mathbf{3 1}$ & $\mathbf{3 7}$ & $\mathbf{9 1}$ & $\mathbf{1 9 9}$ & $\mathbf{3 6 8}$ \\
\hline
\end{tabular}

Quadro 10 Evolução dos Objetos investigados nas publicações do período analisado

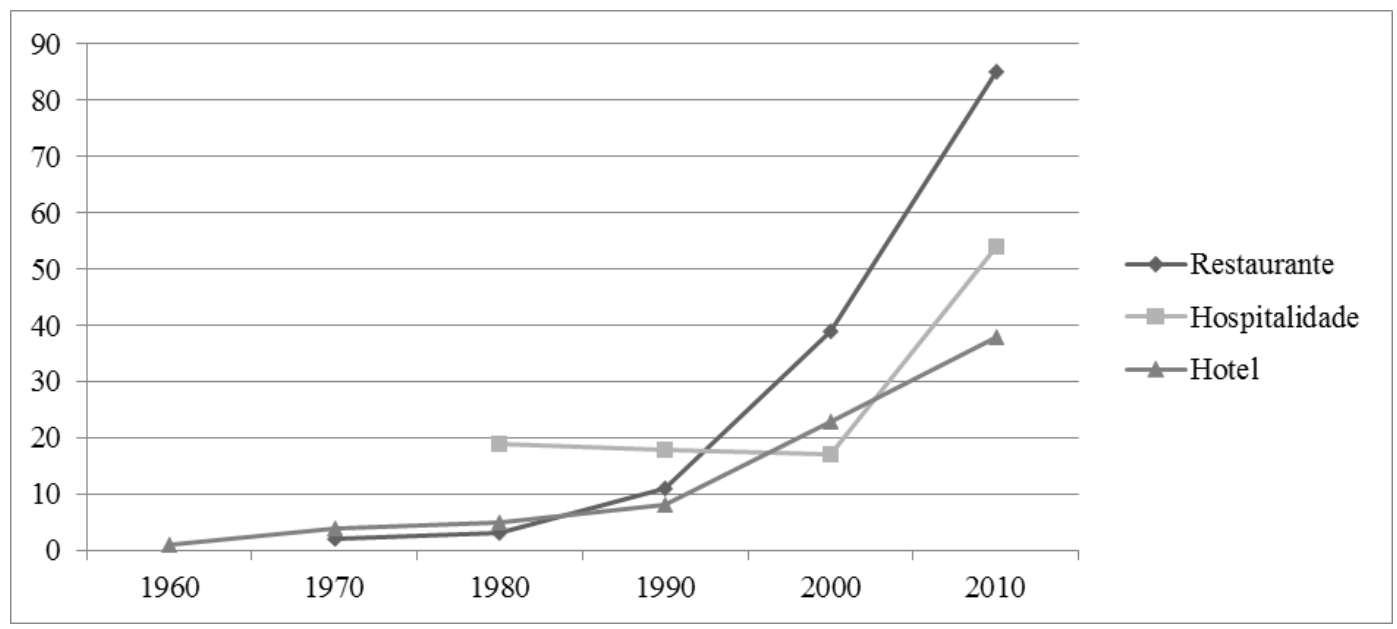

Figura 4 - Evolução dos Objetos mais estudados

Os resultados mostram que a área de Recursos Humanos foi a mais estudada em todo o período selecionado, desde a década de 1960 até o ano de 2015. Podemos perceber, nesse caso, uma eminente inquietação dos pesquisadores quanto às questões voltadas para as pessoas que atuam em empresas de alimentos e bebidas, tais como os restaurantes, os hotéis e outras instituições dentro da área da hospitalidade. Tal evidência corrobora as inferências de Barrows (2008) de que a eficiência das instituições desse setor está diretamente relacionada ao nível de gerenciamento dos recursos humanos. O autor enfatiza que os gerentes de alimentos e bebidas enfrentam grandes desafios no gerenciamento de recursos humanos, devido à escassez de trabalho em algumas regiões e pelo fato de o setor não ser uma opção de carreira atraente. Esse resultado reforça, também, os preceitos de Davis et al. (2012) e Barrows (2008), que destacam o papel do gerente de alimentos e bebidas como corresponsável pelo sucesso das organizações onde atua. Assim como as responsabilidades dos demais colaboradores no cumpri- mento dos objetivos da empresa. A quantidade e a evolução das publicações da área de Recursos Humanos ao longo do tempo demonstram que a maioria das indagações no setor de alimentos e bebidas está mais direcionada aos fatores internos das organizações do que as interações do negócio com as variáveis externas. Questões ligadas à percepção do cliente quanto ao atendimento e ambiente do negócio foram despertadas duas décadas mais tarde.

Depois de Recursos Humanos, a segunda área de maior interesse nesses estudos foi Atendimento e ambiente. Apesar de as primeiras publicações dessa amostragem surgirem a partir da década de 1980, houve um aumento significativo a partir do ano 2000. Após a consolidação dos estudos de recursos humanos, voltados às questões intraorganizacionais, os pesquisadores iniciaram pesquisas para entender melhor a percepção do cliente quanto ao atendimento e ao ambiente oferecido pelos estabelecimentos de alimentos e bebidas. Esse resultado ressalta os objetivos traçados por Davis et al. (2012) para o gerenciamento das empresas de hospitalida- 
de. Segundo os autores, esses objetivos visam garantir que os clientes se sintam bem-vindos no estabelecimento, que as instalações funcionem adequadamente ao convidado, que a operação ofereça serviço enquanto gera lucro e que a expectativa do cliente seja atendida ou superada com o fornecimento dos produtos e serviços da organização. Os autores destacam, ainda, a necessidade de se criar um instrumento que possa obter as avaliações dos clientes e utilizá-las para desenvolver as melhorias necessárias ao negócio. $\mathrm{O}$ surgimento e a evolução dos estudos nessa área indicam um aumento da preocupação dos pesquisadores quanto à percepção e avaliação do cliente em relação aos produtos e serviços oferecidos pelas organizações de alimentos e bebidas.

Em paralelo aos estudos de atendimento e ambiente surgem pesquisas direcionadas ao Conceito do Negócio de alimentos e bebidas. Essa área, assim como Atendimento e ambiente, apresenta publicações desde a década de 1980 com aumento significativo a partir do ano 2000. Os resultados dessa área sustentam os preceitos de Davis et al. (2012) sobre o processo de planejamento nas empresas de alimentos e bebidas. Segundo os autores, as organizações precisam definir políticas que envolvam diversas áreas da instituição, como finanças, marketing e operações. Nessas áreas são desenvolvidas atividades de previsão de lucros e restrição de custos, identificação e definição de mercados-alvos e concorrentes e definição de métodos e operações das empresas. Os resultados apoiam, também, as premissas de Davis et al. (2012) e Riley (2005) sobre as pressões sofridas pelo departamento de alimentos e bebidas. Essas pressões podem ser externas, advindas de mudanças políticas, econômicas, sociais e tecnológicas; e internas, como dificuldades enfrentadas com perecibilidade e desperdício de alimentos, furtos, absenteísmo, entre outros. Os resultados reforçam, ainda, as inferências de Barrows (2008) sobre outras questões que influenciam o setor de alimentos e bebidas, como demografia, aumento de renda das famílias e internacionalização da cozinha.

\section{CONCLUSÕES}

O principal objetivo deste estudo foi identificar, por meio de uma pesquisa bibliográfica feita com publicações disponíveis nas bases de dados EBSCO e Food and Beverage Management Commons, os estudos correlatos com as áreas de gestão no setor de alimentos e bebidas. Teve, também, o objetivo de quantificar, de forma longitudinal, o número de publicações encontradas por área da administração.

Os resultados mostraram que o número de publicações vem aumentando exponencialmente desde os primeiros artigos encontrados na década de 1960. Destaque para os turns das décadas de 1980, 2000 e 2010, que apresentaram em seus respectivos decênios mais que o dobro de publicações que todos os decênios anteriores somados. Os resultados também revelaram a existência de 18 áreas da administração de empresas, sendo que a mais abordada nos estudos de todo o período em questão é a de Recursos Humanos. Em seguida, aparecem as áreas de Atendimento e ambiente e Conceito do Negócio. Por outro lado, os resultados apontaram, ainda, que áreas como Marketing, Qualidade e Gestão de Estoques ainda são pouco estudadas. Dentre os 21 objetos escolhidos pelos pesquisadores, o Restaurante foi o que mais apareceu nas publicações do período investigado, seguido por Hospitalidade e Hotel. Contudo, empreendimentos como Pousadas, Cafeterias, Bares e Catering foram pouco abordados e podem ser alvos de novos estudos no futuro.

Uma das limitações deste estudo foi a utilização de apenas duas bases de dados para a seleção das publicações relacionadas com as palavras-chaves pesquisadas. Sugere-se que, para novas pesquisas a serem realizadas nos mesmos moldes deste estudo, sejam feitas as buscas em mais bases de dados para aumentar a abrangência da 
amostra de publicações relacionadas ao tema. Contudo, alertamos, também, sobre o cuidado na replicabilidade deste estudo em outras bases de dados, visto que alguns periódicos podem não dispor das publicações mais antigas na versão digital. Todavia, espera-se que este estudo tenha contri-

\section{REFERÊNCIAS}

Barrows, C. W. (2008). Food and beverage management. In Brother Ton, B., \& Wood, R. C. (Eds.), The Sage handbook of hospitality management (pp.421-442). Londres, UK: Sage.

Boccato, V. R. C. (2006). Metodologia da pesquisa bibliográfica na área odontológica e o artigo científico como forma de comunicação. Revista de Odontologia da Universidade Cidade de São Paulo, 18(3), 265-274.

Booth, W. C., Colomb, G. G., \& Williams, J. M. (2005). A arte da pesquisa (3a ed.). São Paulo, SP: Martins Fontes.

Botelho, L. L., R. Cunha, C. C. A., \& Macedo, M. (2011). O Método da revisão integrativa nos estudos organizacionais. Gestão e Sociedade, 5(11).

Davis, B., Lockwood, A., Alcott, P., \& Pantelidis, I. (2012). Food and beverage management (5a ed.). New York, NY: Routledge.

Figueiredo, N. (1990). Da importância dos artigos de revisão da literatura. Revista Brasileira de Biblioteconomia e Documentação, 23(1/4).

Lima, T. C. S., \& Mioto, R. C. T. (2007). Procedimentos metodológicos na construção do conhecimento científico: a pesquisa bibliográfica. Revista Katálysis, 10 (esp). buído, de forma seminal, para mapear a área, oferecendo indicadores que sirvam de subsídio e direcionamento para novas pesquisas que venham a auxiliar ainda mais no entendimento da administração de empresas com ênfase na gestão em alimentos e bebidas.

Marietto, M. L., \& Maccari, E. A. (2015). Estudos da estratégia como prática na perspectiva estruturacionista: exemplo de contribuição metodológica. Revista Ibero-Americana de Estratégia-RIAE, 14(1).

Minayo, M. C. (2001). Ciência, técnica e arte: o desafio da pesquisa social. In Minayo, M. C. (Org.), Pesquisa social: teoria, método e criatividade. Petrópolis, RJ: Vozes.

Mota, A. M. G., Ferreira, A. C. V. V., Wada, E. K., \& Santos, M. F. (2014). Turismo de aventura acessível. Podium Sport, Leisure and Tourism Review, 3(1).

PizzanI, L., Silva, R. C., Bello, S. F., \& Hayashi, M. C. P. I. (2012, julho a dezembro). A arte da pesquisa bibliográfica na busca do conhecimento. Revista Digital de Biblioteconomia e Ciência da Informação, 1(1), 53-66.

RILEY, M. (2005). Food and beverage management: a review of change. International Journal of Contemporary Hospitality, 17(1), 88-93.

Sá-Silva, J. R., Almeida, C. D., \& Guindani, J. F. (2009). Pesquisa documental: pistas teóricas e metodológicas. Revista Brasileira de História \& Ciências Sociais, 1(1), 1-15.

Serra, F. A. R., Ferreira, M. P., Pereira, M. F., \& LISSONI, J. (2008). Evolução da pesquisa em RBV: um estudo dos últi- 
mos EnANPAD's. Revista Brasileira de Estratégia, 1(1).

Tews, M. J., \& HOOF, H. B. (2011). In favor of hospitality-management education. FIU Review, 29(2).

Treinta, F. T., Filho, J. R. F., SANT'ANNA, A. P., \& Rabelo, L. M. (2014). Metodologia de pesquisa bibliográfica com a utilização de método multicritério de apoio à decisão. Production, 24(3), 508-520.

Universidade Anhembi Morumbi. (2016). Pós-graduação stricto sensu Mestrado Profissional: gestão em alimentos e bebidas $(A \& B)$. Recuperado em 16 de maio de 2016, de http://portal.anhembi.br/mestradoprofissio nal/

Wood, R. C. (2007). Future of food and beverage management research. Journal of Hospitality and Tourism Management, 14(1). 